\title{
Generation and Propagation of Chloride-Pits on Rotating Stainless Steel Electrode in Acid Solution
}

\author{
By Norio Sato*, Tokio Nakagawa*, \\ Kiyokatsu Kudo* and Masao Sakashita*
}

\begin{abstract}
Anodic pit generation and pit growth of stainless steel at constant potential in a sulphuric acid solution containing chloride ion have been investigated by use of a rotating electrode. The electrode rotation makes the generation and growth of pits very stable and enables the pit generation frequency and the pitting current from a single pit to be measured potentiostatically. The pits break out at constant frequency and the rate of pit generation is a linear function of the potential, suggesting that the pit generation proceeds electromechanically rather than electrochemically. An incubation time, probably associated with the initial adsorption of chloride ion, is found before the linear pit generation commences, which differs from the induction time for opening an initial pit.

The pitting current is in proportion to the area of pit mouth with the current density $\left(8 \mathrm{~A} / \mathrm{cm}^{2}\right)$ which is independent of the potential, indicating that the mass transfer is rate-limiting. The pit grows following a parabolic law and the rate constant is an exponential function of the potential, from which a pit model is proposed in which the transpassive film continuously forming and breaking down on the inner pit surface assumes a high electric field.
\end{abstract}

(Received August 14, 1971)

\section{Introduction}

The pitting corrosion of passivated metals consists of two apparently different processes, nucleation and growth of pits. The nucleation (or generation) of pits is a random process and is related to the local breakdown of the passivating film on the surface. It is therefore sensitive to structural and compositional defects (microscopic imhomogeneity) in the passive film or in the metal surface layer, defects which may exist constitutionally or arise from adsorption of chloride ions. On the other hand, the pit growth proceeds by the metal dissolution from the pit and requires condensation of aggressive ions such as chloride and hydrogen ions in the pit (macroscopic imhomogeneity) to break the passive film continuously or to inhibit the formation of passive films on the inner pit surface ; characteristically, it is the pitting dissolution current itself which produces and maintains the macroscopic imhomogeneity necessary to continue the pit growth.

The pit nucleation (or generation) is frequently characterized by a critical pitting potential, defined as the least positive potential at which pits can be

* Electrochemistry Laboratory, Corrosion Research Center, Faculty of Engineering, Hokkaido University, Sapporo, Japan.

(1) Ja. M. Kolotyrkin : Corrosion, 19(1963), 261t.

(2) H. P. Leckie and H.H. Uhlig : J. Electrochem. Soc., 113 (1966), 1262.

( 3 ) K. J. Vetter : Ber. Bunsenges. Phys. Chem., 69 (1965), 683; K. J. Vetter and H.H. Strehblow : Ber. Bunsenges. Phys. Chem., 74 (1970), 449, 1024.

(4) H. Kaesche : Z. Phys. Chem. (N. F.), 34 (1962), 87; 26(1960), 138.

(5) T. P. Hoar : Corrosion Sci., 7 (1967), 341.

(6) D. A. Vermilyea : J. Electrochem. Soc., 118 (1971), 529

(7) W. Schwenk : Corrosion Sci., 3(1963), 107; Corrosion, 20 $(1964), 129 \mathrm{t}$.

(8) H. Rickert and G. Holzäpfel : Werk. u. Korr., $17(1966), 376$; Ber. Bunsenges. Phys. Chem., 70 (1966), 171.

(9) G. Herbsleb and H. J. Engell : Z. Phys. Chem., 215 (1960), 167. generated or grown, and an induction time for pit initiation, defined as the time required before an initial pit breaks out at constant potential after introduction of chloride ions. Some investigators ${ }^{(1) \sim(6)}$ have discussed the pitting potential as the equilibrium potential of the reaction responsible for pit nucleation, while other investigators ${ }^{(7)(8)}$ have rather attributed it to the pit propagation process. Similarly, the induction time has frequently been discussed as corresponding to the rate of pit nucleation, although strictly there remains a problem whether it can be related directly to the pit nucleation kinetics.

If we consider the pit generation as a random process, the measurements of the frequency (or rate) of pit generation would be important in understanding the pit generation kinetics. Recent studies ${ }^{(9) \sim(16)}$ have shown that during pitting at constant potential the number of pits either increases with time or remains almost constant, depending on the time and the potential. In this paper the pit generation frequency is measured and compared with the induction time for pit initiation.

For the pit propagation previous investigations have presented a number of results, which are mainly concerned with the time variation of pitting dissolution current $^{(9)(15)(17) \sim(20)}$ and the growth of pit radius ${ }^{(9)(13)}$ (15) (17). Generally, the total pitting dissolution current at constant potential is experimentally given by

(10) Z. Szkarska-Smialowska and M. Janik-Czachor : Brit. Corrosion J., 4 (1969), 138.

(11) J. Tousek : Collection Czechoslov. Chem. Commun., 35 (1970), 774; Werks. u. Korr., 21 (1.970), 21.

(12) V. Ashworth, P.J. Boden, J. S. LI. Leach and A. Y. Nehru : Corrosion Sci., 10 (1970), 481.

(13) I. L. Rosenfeld and I.S. Danilov: Corrosion Sci., 7 (1967), 129.

(14) I. Garz., H. Worch and W. Schatt : Corrosion Sci., 9 (1969), 71.

(15) G. Herbsleb : Werk. u. Korr., 17 (1966), 649.

(16) N. Ohtani, K. Aihara and S. Takamoto : J. Japan Inst. Metals, 33 (1969), 377. 
$I=i n=t^{x}$, where $t$ is the time after opening an initial pit and $n$ the number of pits at time $t$. The pitting dissolution current for a single pit is also given by $i=k$ $t^{y}$, with $y$, for instance, $y=0.5^{(13)}$ in stagnant solution. For the growth of pit radius recent work has given $R=K t^{z}$, with exponent $z$, for instant, $z=0.5 \sim 1.0^{(13)}$ (15)(21). Furthermore, investigations of pit morphology ${ }^{(13)(16)(18)(19)(21)(22)}$ have shown that, depending on the potential where pitting occurs, the pit grows in the form either of approximate hemisphere or of orientational pits, and that the mouth of pits either is open or is closed, presumably also depending on the potential. The closed pit has on its mouth a thin passivated metal cover with a number of small defects. These previous results are of value but more is needed to understand the mechanism of pitting dissolution. In this paper experimental results on the pitting dissolution current and the pit growth are presented and discussed in relation to the pit propagation process.

The pitting dissolution of stainless steels is a strongly localized phenomenon on which the local concentration of aggressive ions on the metal surface has a definite influence. There has been, however, only a few experiments ${ }^{(23)}$ conducted in high speed flow of solutions or with rotating electrodes. This paper describes an experimental study on the anodic pitting dissolution (pit generation and propagation) of a rotating stainless steel electrode in a sulphuric acid solution containing chloride ions.

\section{Experimental}

A rotating electrode apparatus was used, in which the electrode was rotated by a synchronous motor through an urethan rope belt and scored pulleys at five different speeds, 450, 840, 1450, 2550 and $4750 \mathrm{rpm}$. The cylindrical specimen electrode, outer diameter 10.4 $\mathrm{mm}$, inner diameter $7 \mathrm{~mm}$ and length $8 \mathrm{~mm}$, was fastened by a binding bolt to a polyvinyl chloride electrode holder in which a nickel bar with screw coupling on both ends was pluged. Electrical contact with the specimen was made through the central nickel bar connected to the rotating steel shaft to which a copper brush was attached.

The electrolytic cell was a spherical glass vessel of $500 \mathrm{~mL}$ with a solution inlet on the upside and an outlet on the bottom, and had an auxiliary platinum plate electrode $10 \mathrm{~cm}^{2}$ and a Luggin capillary inserted from its upside.

The electrode potential of the specimen was measured in reference to a saturated calomel electrode, and the anodic polarization was carried out by using a high speed response potentiostat (Nichia HP-E) connected to a current recorder (Hitachi QPD 33).

(17) H. J. Engell and N. D. Stolica : Z. Phys. Chem., (N. F. ), 20 (1959), 113; Arch. Eisenhutt., 30 (1959), 239.

(18) K. J. Vetter and H. H. Strehblow : Ber. Bunsenges. Phys. Chem., 74 (1970), 1024.

(19) T. Yoshii and Y. Hisamatsu : J. Japan Inst. Metals, 35 (1971), $151 ; 34$ (1970), 1207.

(20) T. Suzuki and Y. Kitamura : Denki Kagaku, 38 (1970), 526; Corrosion Engl. Japan, 18 (1969), 100; 17 (1968), 535.
The stainless steel specimen used was a commercial SUS 27 Steel ; C, $0.06 \%$; Cr, 18.48 ; Ni, 8.95 ; Si, 0.56 ; $\mathrm{Mn}, 1.02 ; \mathrm{P}, 0.020$; and $\mathrm{S}, 0.011$. The specimen, mechanically polished with emery papers, was vacuumannealed at $1100^{\circ} \mathrm{C}$ for 10 minutes followed by quenching. The surface was then softly polished again using fine emery paper $(0 / 4)$, and etched in an acid $(6 \%-$ $\mathrm{HNO}_{3} 10.2$ vol $\% 46 \%-\mathrm{HF} 2.2$ vol $\%$, and $\mathrm{H}_{2} \mathrm{O}$ balance) for several minutes immediately followed by washing in a jet of redistilled water. After dried with benzene and acetone, the specimen was fastened to the electrode holder, and the boundary between the specimen and the holder was sealed using a celluloid lacquer (transparent manicure) to expose the specimen surface $1 \mathrm{~cm}^{2}$.

The solutions used in this pitting study were $0.2 \mathrm{M}$ $\mathrm{NaCl}$ plus $0.1 \mathrm{M} \mathrm{Na}_{2} \mathrm{SO}_{4}$ solutions whose $\mathrm{pH}$ values were adjusted by adding small amounts of $\mathrm{H}_{2} \mathrm{SO}_{4}$ at $\mathrm{pH}$ 2.3, $3.0,3.5,4.0$ and 5.0. The solutions used for passivating the specimen were $0.1 \mathrm{M} \mathrm{Na}_{2} \mathrm{SO}_{4}$ solutions of the same $\mathrm{pH}$ as above. These solutions were made of analytical grade regents and redistilled water.

In potentiostatic pitting dissolution experiments, the specimen electrode was passivated at a given potential in $0.1 \mathrm{M} \mathrm{Na}_{2} \mathrm{SO}_{4}$ solution of a given $\mathrm{pH}$ for $1 \mathrm{hr}$, and then the solution was replaced by the test solution $(0.2$ $\mathrm{M} \mathrm{NaCl}+0.1 \mathrm{M} \mathrm{Na}_{2} \mathrm{SO}_{4}$ ) of the same $\mathrm{pH}$. Immediately after the solution replacement the specimen was polarized again at the same given potential to follow the pitting dissolution. The pitting occurred sometime after the potentiostatic polarization started in the chloride solution, which could be observed by a sudden increase in anodic current. The time required before this sudden current increase appeared was measured as the induction time, $\tau_{1}$, for opening the initial pit. The anodic current during the pit growth was measured as the pitting dissolution current.

In addition, microscopic observations were made to measure the form, figure, radius and depth of pits as functions of time. All the measurements were performed at room temperature $\left(23^{\circ} \pm 2^{\circ} \mathrm{C}\right)$ in air-saturated solution.

\section{Results}

\section{Pitting in rotating electrode}

Figure I shows the pitting potential and the induction time as functions of the speed of electrode rotation. Within the amount of scatter, the rotation speed has little influence on the pitting potential and on the induction time for pit initiation in the range of rotation speed examined.

The rotation speed, however, does affect the initial stage of pitting dissolution. As shown in Fig. 2, the pitting began with an initial rise of anodic current

(21) Y.Matsumura and Y. Hisamatsu : 17 th Annual Symposium on Corrosion and Protection in Japan (1970), Extended Abstract, p. 377; T. Yoshii, Y. Hisamatsu and Y. Matsumura : J. Japan Inst. Metals, 35 (1971), 633.

(22) P. Forchhammer and H. J. Engell : Werk .u. Korr., 20 (1969), 1.

(23) I. V. Riskin and A.B.Turkovskaya : Zashch. Metal., 5 (1969), 443 

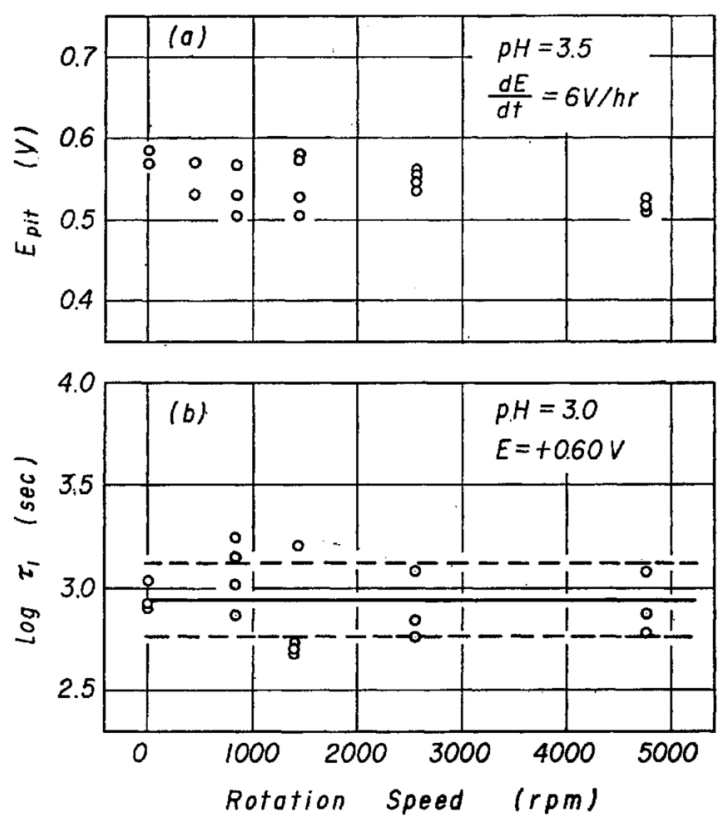

Fig. 1 Effect of rotation speed on the pitting potential (a) and the induction time (b) for opening an initial pit on stainless steel electrode in $0.2 \mathrm{M} \mathrm{NaCl}+0.1 \mathrm{M} \mathrm{Na}_{2} \mathrm{SO}_{4}$ solution. $\quad E_{\text {pit }}$ was measured by potential sweep at $6 \mathrm{~V} / \mathrm{hr}$ from a starting potential $+0.4 \mathrm{~V}$ (SCE) where the specimen, prior measurement, was potentiostatically passivated for 1 hour in the test solution. The specimen for measuring $\tau_{1}$ was passivated for 1 hour at $0.6 \mathrm{~V}$ (SCE) in $0.1 \mathrm{M} \mathrm{Na}_{2} \mathrm{SO}_{4}$ solution of $\mathrm{pH} 3$ before introduction of chloride ion.

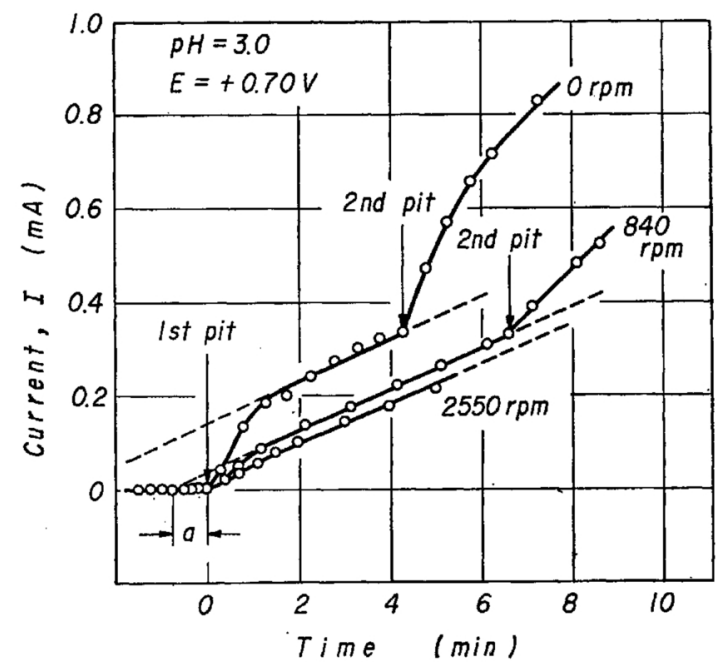

Fig. 2 Time variation of pitting dissolution current at constant potential $(+0.7 \mathrm{~V})$ after opening the initial pit on stationary and rotating stainless steel electrodes in solution of $\mathrm{pH} 3\left(0.1 \mathrm{M} \mathrm{Na}_{2} \mathrm{SO}_{4}+0.2 \mathrm{M} \mathrm{NaCl}\right)$. Specimens were potentiostatically passivated at $+0.7 \mathrm{~V}$ for 1 hour in $0.1 \mathrm{M} \mathrm{Na}_{2} \mathrm{SO}_{4}$ solution of $\mathrm{pH} 3$ prior to introduction of chloride ions.

followed by a steady increase of current which continued until another pit broke out. The initial rise of anodic current was remarkable with the specimen not rotating in stationary solution, while it was only fractional with the rotating electrodes. In the steady pitgrowth the pitting dissolution current increased linearly with time, and we obtain for a single pit,

$$
i=k_{i}(t+a)
$$

where $i$ is the dissolution current, $t$ the time after opening the initial pit, $k_{i}$ the rate of current increase, and $a$ is constant depending on the initial current increase. Constant $k_{i}$ was nearly independent of the rotation speed, and constant $a$, although decreasing remarkably by the electrode rotation, was not affected by the speed of electrode rotation in the range 840 4750 rpm. Another effect of rotation was the deactivation of freshly formed pits.

Figure 3 shows the potentiostatic pitting dissolution current as functions of time. The current-time curve can be seen not to depend very much on the rotation speed but rather to depend on the number of pits being active on the specimen surface.

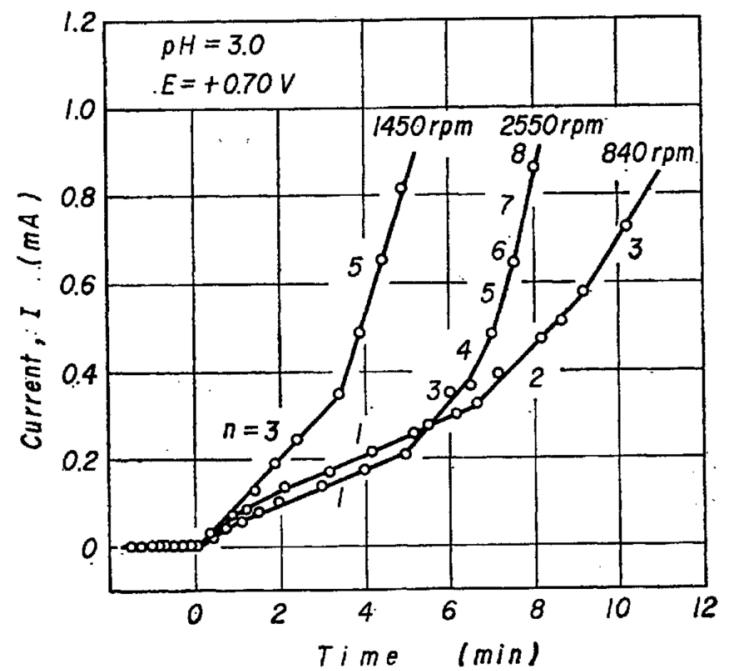

Fig. 3 Time variation of pitting dissolution current at constant potential after opening the initial pit on stainless steel electrode rotating at different speeds in solution of $\mathrm{pH}$ $3\left(0.1 \mathrm{M} \mathrm{Na}_{2} \mathrm{SO}_{4}+0.2 \mathrm{M} \mathrm{NaCl}\right)$. $n$ denotes the number of growing pits.

\section{Pit generation}

In Fig. 3 we have seen that the current-time curve consists of straight line segments. By observing the specimen surface optically, we found that a break in the current-time curve corresponded to a generation of a pit. The current-time curve, therefore, enabled us to measure the induction time for a series of pits successively breaking out, the way of measurements being schematically shown in Fig. 4.

To investigate the kinetics of pit generation, we measured a number of current-time curves in the solution of $\mathrm{pH} 3$ and estimated the induction time for pits one by one in sequence of pit generation. Results obtained are given in Fig. 5. Although the amount of scatter is relatively large, it is evident that the pit generation in average proceeds linearly with time and that the more noble potential results in the larger rate (or frequency) of pit generation. Thus, we obtain for the pit generation

$$
n=k_{p}\left(\tau_{n}-\tau_{0}\right)
$$

where $n$ is the number of pits generated in time $\tau_{n}$ which in turn is the induction time for the $n$-th pit, $k_{p}$ the average pit generation rate (or frequency), and $\tau_{0}$ a constant. 


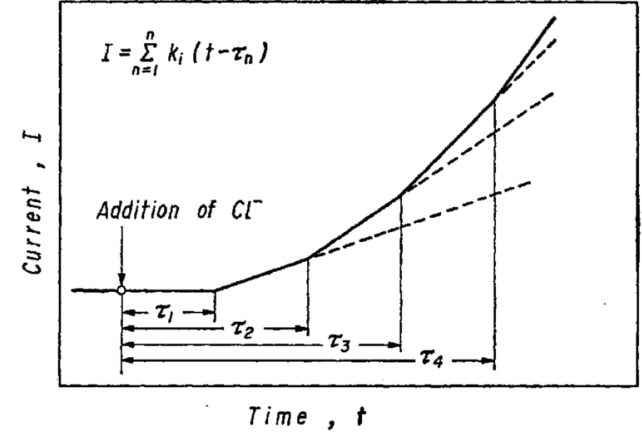

Fig. 4 Schematic representation of current-time curve of potentiostatic pitting dissolution of rotating stainless steel electrode by chloride ions. $\tau_{n}$ denotes the induction time required for the $n$-th pit to break out.
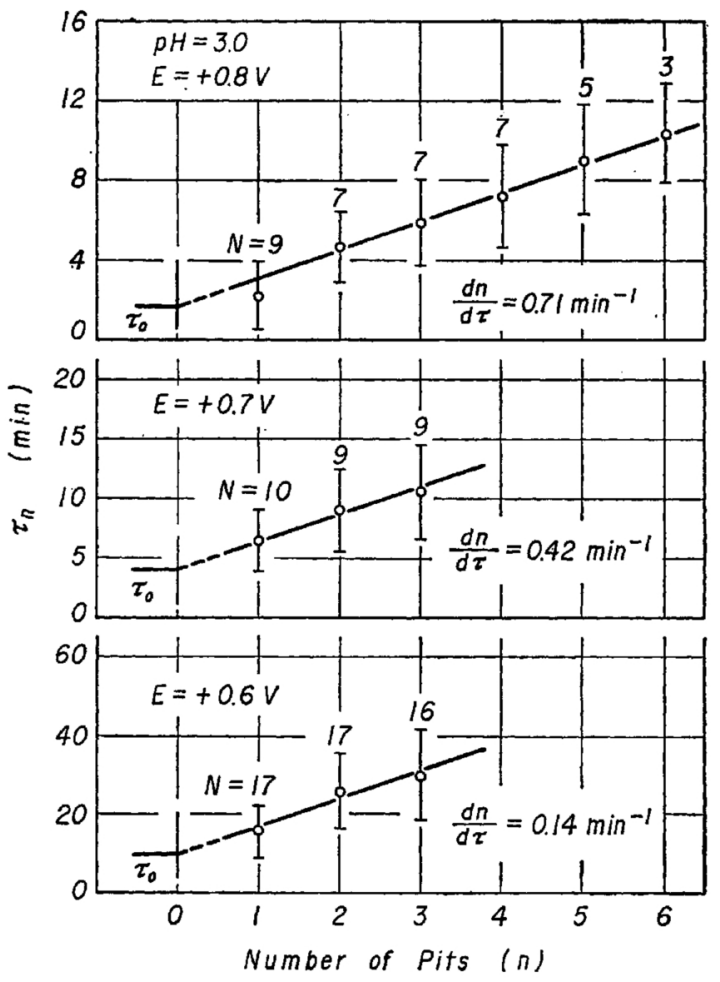

Fig. 5 Induction time $\tau_{n}$ for opening pits in sequence as a function of pit number $n$ at three different potentials in solution of $\mathrm{pH} 3\left(0.1 \mathrm{M} \mathrm{Na}_{2} \mathrm{SO}_{4}+0.2 \mathrm{M} \mathrm{NaCl}\right)$. The open circle is the mean value based on $N$ measurements and the vertical line segment is the standard deviation.

It is found, as shown in Fig. 6, that the rate (or frequency) of pit generation is a linear function of the electrode potential. Thus, we obtain

$$
k_{p}=\beta_{p}\left(E-E_{\mathrm{crit}}\right)
$$

where $\beta_{p}$ is constant, and $E_{\text {crit }}$ is the potential at which the pit generation frequency is expected to be zero; $\beta_{p}=2.8 \mathrm{~V}^{-1} \mathrm{~min}^{-1}$ and $E_{\text {crit }}=+0.552 \mathrm{~V}(\mathrm{SCE})$ for the solution of $\mathrm{pH} 3$.

Furthermore, it is found, as shown in Fig. 7, that the time $\tau_{0}$ depends exponentially on the potential, and we obtain a Tafel relation

$$
E=A_{\tau_{0}}+b_{\tau_{0}} \log \left(1 / \tau_{0}\right)
$$

where $b_{\tau 0}$, the Tafel slope, is evaluated $b_{\tau 0}=270 \mathrm{mV}$ for

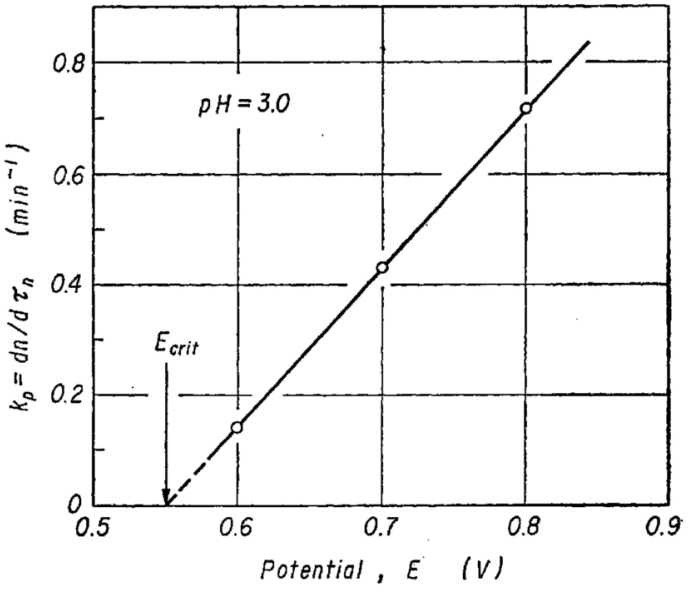

Fig. 6 Effect of the potential on the pit generation rate. The plot is based on the results given in Fig. 7.

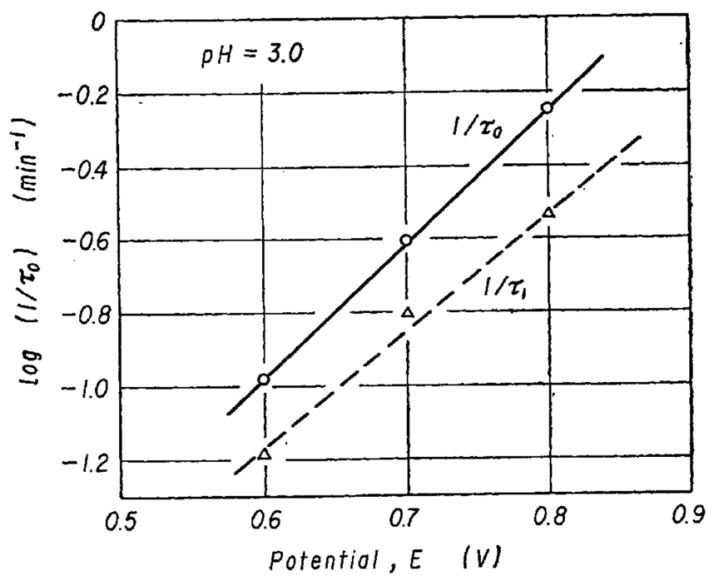

Fig. 7 Effect of potential on the incubation time $\tau_{0}$ for onset of linear pit generation. Plot is based on the results given in Fig. 9 and the broken line indicates $\tau_{1}$ for comparison.

the solution of $\mathrm{pH} 3$. Note that, as compared in Fig. 7, $\tau_{0}$ differs from the induction time $\tau_{1}$ and indicates the time at which the linear pit generation commences, which we call here the incubation time.

\section{Pitting dissolution current}

In Fig. 3 we have shown that the dissolution current from steadily growing pits increases linearly with time, and its rate of increase depends on the number of growing pits. From some seventy current-time curves measured, we estimated the increase rate of the dissolution current in potentiostatic pitting as functions of the number of active pits, the rotation speed, and the electrode potential. Examples of the results are given in Fig. 8. The rate of the current increase is not affected by the rotation speed but is proportional to the number of growing pits. We therefore obtain

$$
\frac{d I}{d t}=k_{i} n
$$

where $k_{i}$ is the rate of the current increase for a single pit. It is found, as shown in Fig. 9, that $k_{i}$ increases almost exponentially with the potential and its Tafel slope is about $400 \mathrm{mV}$. 


\section{Pit form}

From microscopic observations we found that almost all the pits formed were approximately hemispherical (Photo. 1). At higher potentials the pits were always open, while at lower potential - for instant $+0.6 \mathrm{~V}$ (SCE) - the closed pits were formed which had a thin

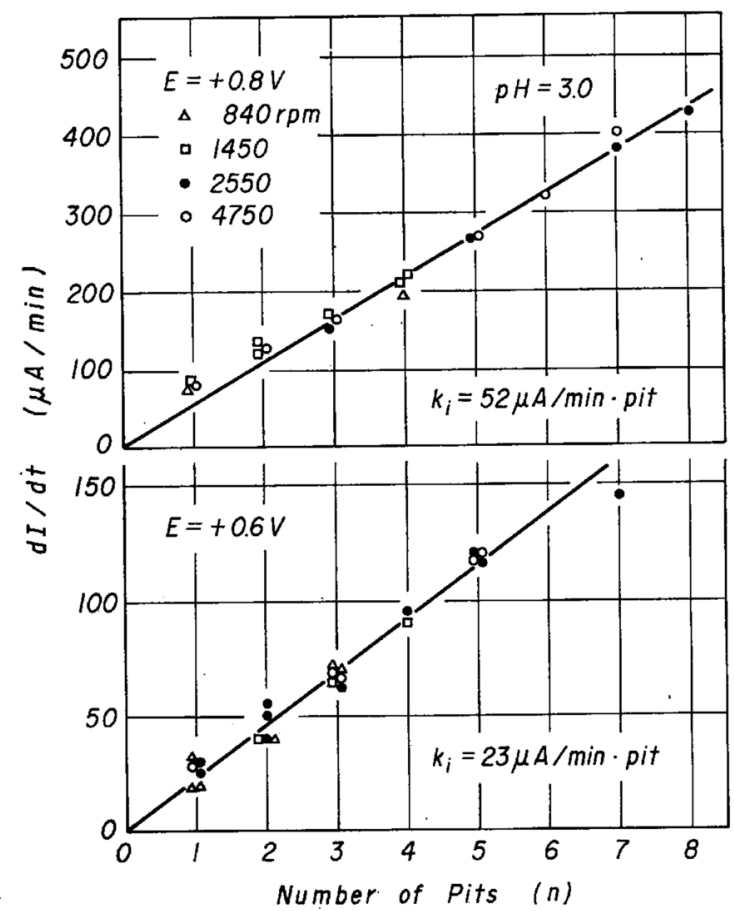

Fig. 8 The rate of current increase at constant potential during pit growth as a function of the number of growing pits on rotating stainless steel electrode in solution of $\mathrm{pH} 3$ (0.1 $\mathrm{M} \mathrm{Na}_{2} \mathrm{SO}_{4}+0.2 \mathrm{M} \mathrm{NaCl}$ ). metal cover with a relatively large hole at its centre and a number of small holes distributed at random.

Furthermore, we measured the radius $R$ and depth $H$ for a number of pits formed at various potentials. Some results are given in Fig. 10, from which it is clear that the pits are not exactly hemispherical and increase in the ratio $H / R$ as they grow.

\section{Pit growth}

To follow the steady pit growth we measured the radius of pits as functions of time after they broke out. Figure 11 gives an example, from which it is apparent that the rate of the pit growth at constant potential is almost independent of the speed of electrode rotation and that the pit radius in average increases following a nearly parabolic scheme.

$$
R=k_{R} \sqrt{t}
$$

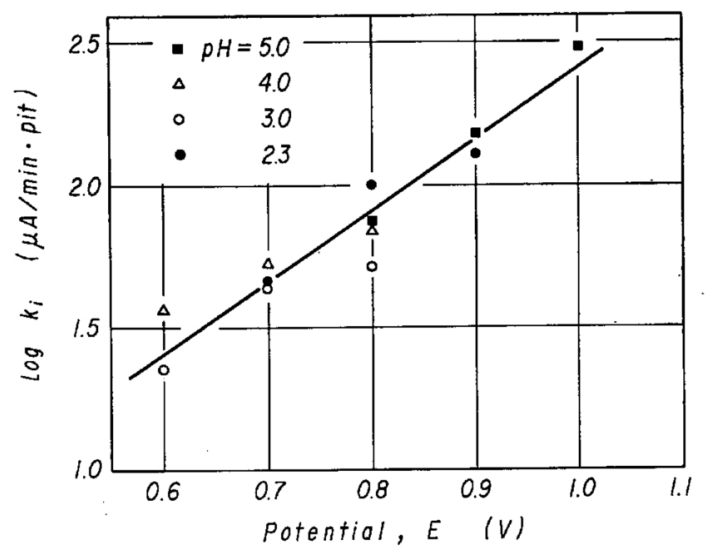

Fig. 9 Potential dependence of $k$, $k_{i}=[d(\partial I / \partial t) / d n]_{E}$.

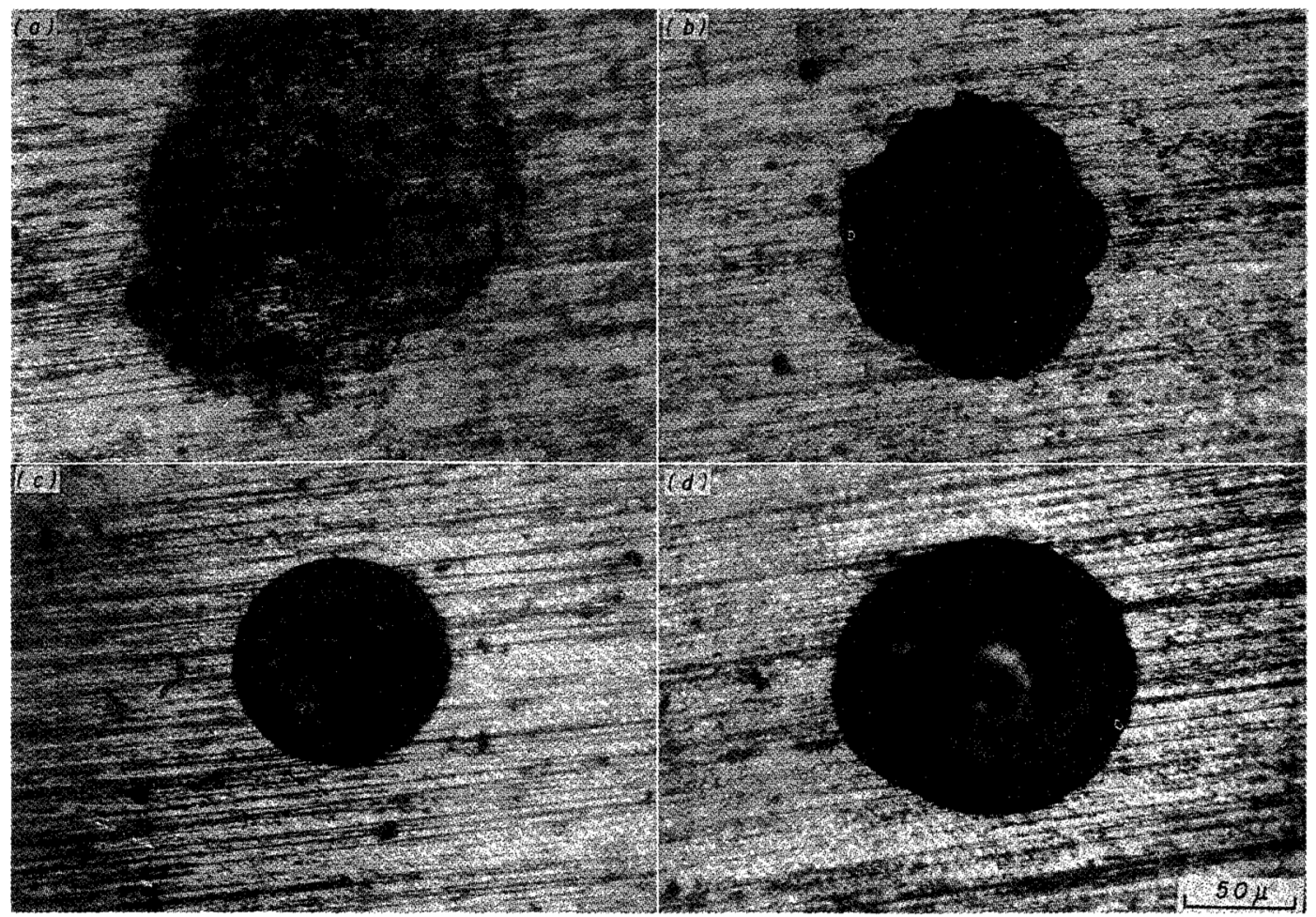

Photo. 1 Microscopic view of pits formed on rotating stainless steel electrode in solution of $\mathrm{pH} 3\left(0.1 \mathrm{M} \mathrm{Na}_{2} \mathrm{SO}_{4}+0.2 \mathrm{M}\right.$ $\mathrm{NaCl}$. (a) $+0.60 \mathrm{~V}, 26.5 \mathrm{~min}, 1450 \mathrm{rpm}$ (b) $+0.70 \mathrm{~V}, 15.8 \mathrm{~min}, 840 \mathrm{rpm}$ (c) $+0.80 \mathrm{~V}, 6.2 \mathrm{~min}, 1450 \mathrm{rpm}$ (d) $+0.90 \mathrm{~V}, 3.5 \mathrm{~min}, 840 \mathrm{rpm}$ 


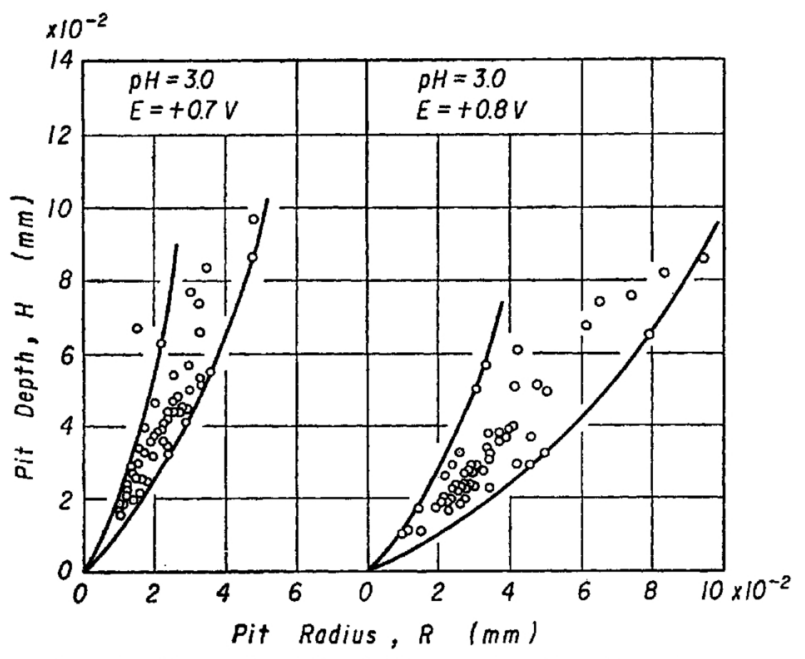

Fig. 10 The ratio of depth $H$ to radius $R$ as functions of pit radius $R$ for a number of pits formed at $+0.7 \mathrm{~V}$ and $+0.8 \mathrm{~V}$ in solution of $\mathrm{pH} 3(0.1 \mathrm{M}$ $\mathrm{Na}_{2} \mathrm{SO}_{4}+0.2 \mathrm{M} \mathrm{NaCl}$ ).

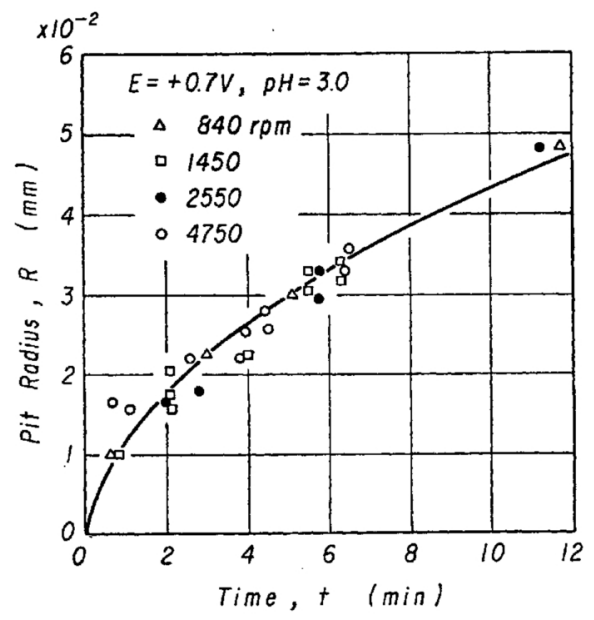

Fig. 11 Growth in radius of pit mouth at constant potential on the rotating stainless steel electrode in solution of $\mathrm{pH} 3\left(0.1 \mathrm{M} \mathrm{Na}_{2} \mathrm{SO}_{4}\right.$ $+0.2 \mathrm{M} \mathrm{NaCl}$ )

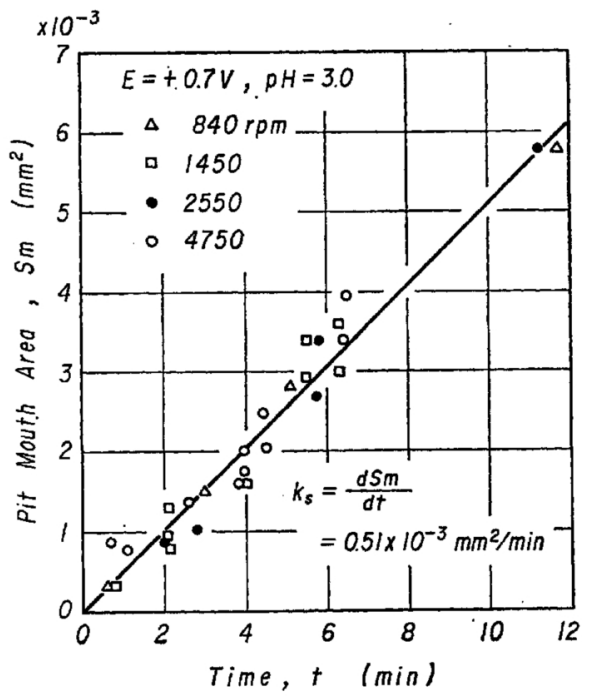

Fig. 12 Growth in area of pit mouth at constant potential on the rotating stainless steel electrode in solution of $\mathrm{pH} 3(0.1 \mathrm{M}$ $\mathrm{Na}_{2} \mathrm{SO}_{4}+0.2 \mathrm{M} \mathrm{NaCl}$ ).
This relation can be examined in Fig. 12, where the area of pit mouth, $S_{m}=\pi R^{2}$, is plotted against time. From this result together with other results at different potentials, we obtain

$$
S_{m}=k_{s} t
$$

where $k_{s}$ is the rate of pit growth in area of the pit mouth. This is in agreement with the result reported by Rosenfeld and Danilor ${ }^{(12)}$ who made measurements with artificial pits.

It is also found, as shown in Fig. 13, that $k_{s}$ is an exponential function of the potential with a Tafel slope about $400 \mathrm{mV}$.

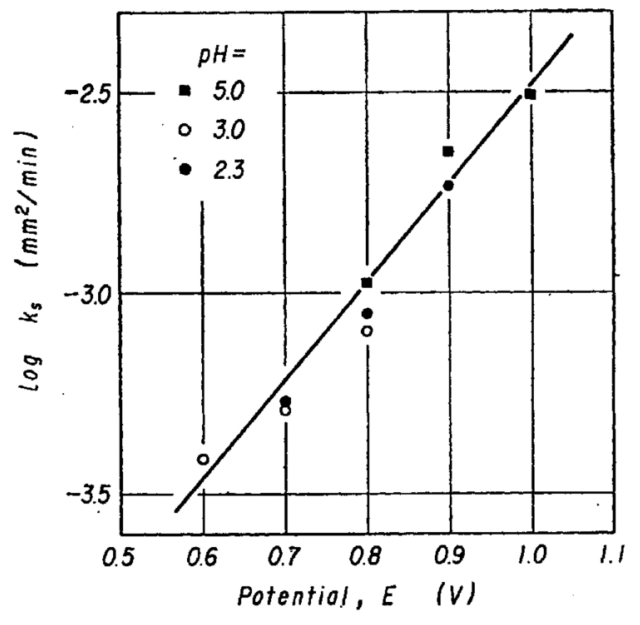

Fig. 13 Potential dependence of $k_{s}$, $k_{s}=\left(\partial S_{m} / \partial t\right)_{E}$

\section{Discussion}

\section{Pitting in rotating electrode}

The specimen electrode rotating at $450 \sim 4700 \mathrm{rpm}$ in the test solution induces turbulent flow of solution on the electrode surface, since its Reynolds number $R e=2-40 \times 10^{3}$ is beyond the laminar flow region. The test solution contained a saturated amount of dissolved oxygen, which however would have little effect on the pitting dissolution current in the potential range examined because practically no cathodic reduction of oxygen occurs at potentials more positive than $+0.6 \mathrm{~V}(\mathrm{SCE})^{(24)}$.

From Fig. 1, which shows no influence of high speed flow of solution both on the pitting potential and on the induction time for pit initiation, we see that any mass transfer does not control the pit nucleation process and hence that the pit generation is a process which occurs in the passivating film itself.

As shown in Fig. 2, the pit growth in the initial stage was more remarkable in stagnant solution than in moving solution, and the stability of freshly formed pits depended on mass transfer in the solution outside the pits to greater extent in stagnant solution than in turbulent flow. In the steady pit growth region, however, the pitting dissolution current increased

(24) G. Okamoto, T. Shibata and N.Sato : Annual Rep. The Asahi Glass Foundation for the Contribution to Industrial Technology, 15 (1969), 207. 
steadily at a constant rate which, although depending on the potential, was not affected by the speed of electrode rotation (Figs. 2, 3 and 8 ). It is therefore apparent that the stability and the growth rate of pits are no longer dependent on the solution flow outside pits but rather controlled by the concentrated solution inside pits upon which the electrode rotation would have little effect.

\section{Pit generation}

In Fig. 4 we have shown that the rotation of specimen electrodes enables us to measure not only the induction time for opening the initial pit but also the rate of pit generation by simply observing the time variation of anodic current.

We have seen in Fig. 5 that the number of pits increases linearly with time at constant potential. The rate of pit generation is thus given from eq. (2) by

$$
k_{p}=\left(\partial n / \partial \tau_{n}\right) E .
$$

In recent work $^{(9)(11)(13)}$ stainless steel as well as iron, even in stagnant solution, showed a linear kinetics of pit generation at constant potential, with a few exceptions ${ }^{(9)(10)(12)}$ showing non-linear pit generation probably as a result of the interaction between pits which would become effective with increase of pit density.

It is worth to note that the pit generation rate $k_{p}$ differs from the inverse of the induction time $1 / \tau_{1}$, the rate of opening the initial pit. The relation between them is obtained from eq. (2) as

$$
\frac{1}{\tau_{1}}=\frac{k_{p}}{\left(1+k_{p} \tau_{0}\right)} \text {. }
$$

We thus see that $1 / \tau_{1}$ would approximate the rate of pit generation $k_{p}$, only if $\tau_{0}$ be very close to zero.

In Fig. 5 we have seen that $\tau_{0}$ is the time at which the linear pit generation process might start after introduction of chloride ions. This is the incubation time associated with opening the linear pit generation and may be explained as the time required before the passivating film is in equilibrium or steady state with respect to the adsorption of chloride ion. To explain $\tau_{0}$ we give Fig. 14, which shows schematically the change that would occur in the passivating film. Evidently, we see that $1 / \tau_{0}$ corresponds not to the kinetics of the film breakdown or pit generation but rather to the rate of chloride ion adsorption on or incorporation to the passivating film. As shown in Fig. $7,1 / \tau_{0}$ increased
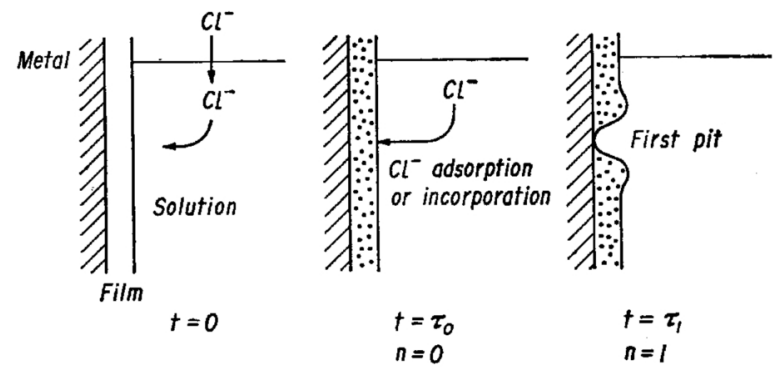

Fig. 14 Scheme for the incubation stage of pitting. exponentially with the potential in agreement with a Tafel relation, suggesting that an electrochemical process such as chloride ion adsorption is responsible for $1 / \tau_{0}$.

Furthermore, from the fact that the rate of frequency of pit generation $k_{p}$ depends linearly with the potential (Fig. 6), we may see that the process which controls the rate of pit generation, i.e. the frequency of film breakdown, is not an electrochemical reaction but rather an electrical process such as a mechanical film breakdown due to the electrostriction pressure, as previously proposed by one of the authors ${ }^{(25)}$. We need, of course, further experiments before clarifying the mechanism of pit generation and film breakdown. However, any further study on the kinetics of pit generations should take into account the difference in physical meaning between the induction time $\tau_{1}$, the incubation time $\tau_{0}$, and the pit generation rate $k_{p}$ described above.

\section{Pitting dissolution current}

In Photo. 1 we have shown that all the pits except those formed at $+0.6 \mathrm{~V}$ are of the open type and their mouth are approximately circular. At $+0.6 \mathrm{~V}$ the pits were of the closed type. The closed pits on stainless steels have of ten been observed by other workers ${ }^{(13)(19)}$ (22) at potentials rather close to the critical pitting potential. From examining the potentials for open and closed pits we see that there might be a boundary potential separating the potential regions for the two types of pit, as suggested by Hoar ${ }^{(5)}$ and Bianchi ${ }^{(26)}$. We here discuss the pitting dissolution current from the open pit.

During the steady growth the pitting dissolution current from a single pit was a linear function of time (Figs. 4 and 8 ) and the area of the pit mouth increased linearly with time (Fig. 12). We see therefore that the pitting dissolution current is in proportion to the area of pit mouth.

The metal ion transfer across the pit mouth may be given by the flux

$$
\Phi=k S_{m}
$$

where $k$ is the flux density of transferring metal ion at the pit mouth. Furthermore, the material balance with respect to the metal ion gives

$$
\Phi=(i / z F)-C_{M}(d V / d t)
$$

where the first term represents the rate of metal dissolution inside the pits and the second the rate of accumulation of metal ion in the pit, $C_{M}$ being the concentration of metal ion in the pit and $V$ the volume of pit. Since $i=(z F \rho / M)(d V / d t)$, we obtain from eq. (11)

$$
\Phi=(i / z F)\left(1-M C_{M} / \rho\right)
$$

where $M$ and $\rho$ are the molecular weight and the density of the metal, respectively. Note that we have here assumed that the concentration of metal ion in the pit, $C_{M}$, is unchanged during the pitting dissolution ;

(25) N. Sato : J. Japan Inst. Metals, 34 (1970), 615.

(26) G. Bianchi, A. Cerquetti, F. Mazza and S. Torchio : Corrosion Sci., 10 (1970), 19. 
$C_{M}$ would be very large and close to the saturated concentration for metal salts.

From eqs. (10) and (12),

$$
i=\frac{z F k S_{m}}{1-\left(M C_{M} / \rho\right)}=i_{0} S_{m}
$$

and hence

$$
i_{0}=\frac{z F k}{1-\left(M C_{M} / \rho\right)}
$$

From our experiments we estimate the current density $i_{0}$ at the pit mouth. From eqs.(1) and (7), and neglecting the term $k_{i} \cdot a$ in eq. (1) because it vanishes at high speed flow of solution, we obtain,

$$
i=\left(k_{i} / k_{s}\right) S_{m}
$$

and hence

$$
i_{0}=k_{i} / k_{s} \text {. }
$$

Table 1 summarizes $k_{i}, k_{s}$, and $i_{0}$ evaluated from our experiments. As an average, we obtain

\begin{tabular}{|c|c|c|c|c|}
\hline $\begin{array}{l}\text { Potential } \\
\text { (V) }\end{array}$ & $\begin{array}{c}\text { Solution } \\
\text { (pH) }\end{array}$ & $\begin{array}{c}k_{i}=d(d I / d t) / d n \\
(\mu \mathrm{A} / \mathrm{min} \cdot \mathrm{pit})\end{array}$ & $\begin{array}{c}k_{s}=d S_{m} / d t \\
\left(\mathrm{~mm}^{2} / \mathrm{min}\right)\end{array}$ & $\left(\mathrm{A} / \mathrm{cm}^{2}\right)$ \\
\hline 1.0 & 5.0 & 305 & $3.10 \times 10^{-3}$ & 9.83 \\
\hline 0.9 & $\begin{array}{l}5.0 \\
2.3\end{array}$ & $\begin{array}{l}150 \\
131\end{array}$ & $\begin{array}{l}2.25 \\
1.85\end{array}$ & $\begin{array}{l}6.68 \\
7.08\end{array}$ \\
\hline 0.8 & $\begin{array}{l}5.0 \\
4.0 \\
3.0 \\
2.3\end{array}$ & $\begin{array}{r}76.5 \\
72.0 \\
52.0 \\
100\end{array}$ & $\begin{array}{l}1.05 \\
-\overline{0.80} \\
0.90\end{array}$ & $\begin{array}{r}7.28 \\
6.50 \\
11.1\end{array}$ \\
\hline 0.7 & $\begin{array}{l}4.0 \\
3.0 \\
2.3\end{array}$ & $\begin{array}{l}54.0 \\
43.6 \\
46.0\end{array}$ & $\begin{array}{l}\overline{0.51} \\
0.52\end{array}$ & $\begin{array}{l}\overline{8.46} \\
8.84\end{array}$ \\
\hline 0.6 & $\begin{array}{l}4.0 \\
3.0\end{array}$ & $\begin{array}{l}37.0 \\
23.0\end{array}$ & $\overline{0.39}$ & $\overline{5.90}$ \\
\hline
\end{tabular}

Talbe 1 Rate of pitting current increase for a single pit, growth rate in area of pit mouth, and pitting current density at pit mouth.

$$
i_{0}=8 \mathrm{~A} / \mathrm{cm}^{2}
$$

The fact that $i_{0}$ was almost independent of the speed of electrode rotation is readily explained by the turbulent flow which provides a nearly constant thickness of the diffusion layer on the metal surface.

It is remarkable that the current density $i_{0}$ is not affected by the potential and the solution $\mathrm{pH}$. If we assume that the mass transfer of metal ion across the pit mouth is controlled mainly by the diffusion, $k$ in eq. (10) is approximated by $D_{M}\left(C_{M} / \delta\right)$, and from eq. (14) we obtain

$$
C_{M}=\frac{i_{0}}{z F\left(D_{M} / \delta\right)+i_{0}(M / \rho)} .
$$

Rough estimate using $D_{M}=1 \times 10^{-5} \mathrm{~cm} / \mathrm{sec}$ and $\delta=0.001$ $\sim 0.005 \mathrm{~cm}$ gives $C_{M}=4 \sim 20 \mathrm{~mol} / \mathrm{L}$, from which it is suggested that the concentration of metal ion in the pit is in saturation or in super saturation with metal salts. In Fig. 15 the current density $i_{0}$ estimated by other workers $^{(7)(10)(19)}$ is compared with ours. Note that at potentials less positive than $+0.6 \mathrm{~V}$ the closed pits are formed, while the pits formed at more positive potentials are open. At this stage, it is not made clear whether the decrease of $i_{0}$ with descending the potential results from the resistance in mass transfer across the pit cover or from the pitting dissolution mechanism that might differ depending on the potential region.

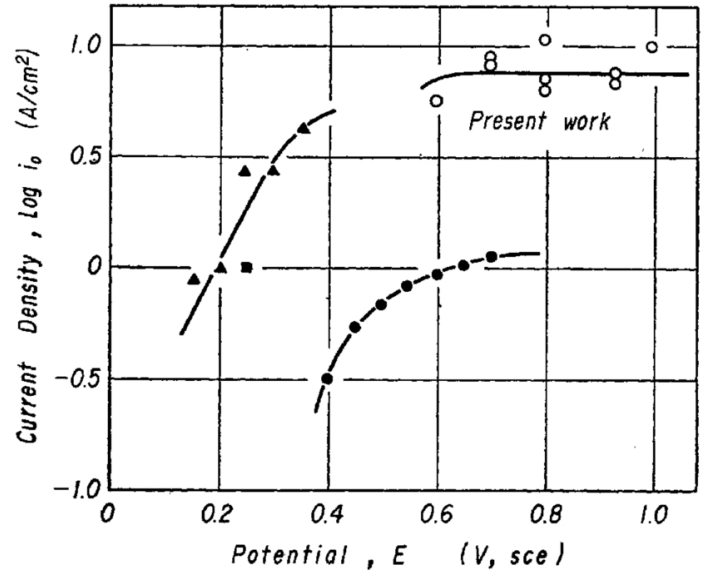

Fig. 15 Pitting current density at pit mouth. O Present work

- $18 \mathrm{Cr}-8 \mathrm{Ni}, 1 \mathrm{~N} \mathrm{H}_{2} \mathrm{SO}_{4}+0.5 \mathrm{M} \mathrm{NaCI}$, stagnant solution by Yoshii and Hisamatsu ${ }^{(19)}$.

- $16 \mathrm{Cr}, 0.7 \mathrm{~N} \mathrm{Na}_{2} \mathrm{SO}_{4}+0.7 \mathrm{~N} \mathrm{NaCl}$, stagnant solution by Szklarska-Smialowska and JanikCzachor ${ }^{(10)}$

- $18 \mathrm{~N}-10 \mathrm{Cr}, 1 \mathrm{~N} \mathrm{H}_{2} \mathrm{SO}_{4}+1 \mathrm{M} \mathrm{NaCl}$, stagnant solution Schwenk ${ }^{(7)}$.

Nevertheless, from our results it is evident that in the potential region where the current density $i_{0}$ is constant the pitting dissolution proceeds in much the same mechanism as that of electropolishing or electrobrightening.

\section{Form of pits}

In Fig. 11 we have shown that the form of pits is not exact hemisphere but changes in the ratio of depth to radius $H / R$ during pit growth. Expressing the volume of a pit as $V=(2 / 3) \pi R^{3} \alpha$, where $\alpha$ is a correction factor for deviation from hemisphere, and assume $\alpha$ to be given by $\alpha=\alpha_{0} R^{x}$, we have $V=(2 / 3) \pi \alpha_{0}$ $R^{(3+x)}$. Since our experiments gave $R=k_{R} t^{0.5}$, we obtain the pit volume as a function of time $V=(2 / 3) \pi \alpha_{0}$ $k_{R}^{(3+x)} t^{(1.5+0.5 x)}$.

The pitting dissolution current from a single pit is therefore given by $i=(z F M / \rho)(d V / d t)=K^{\prime} t^{0.5+0.5 x}$. Comparing this with the experimental results given by $i \approx k_{i} t$, we obtain $x \approx 1$, and hence the correction factor $\alpha$ is nearly proportional to the pit radius. This is in qualitative agreement with the results shown in Fig. 10.

\section{Pit growth kinetics}

In the first approximation we take the radius of the pit mouth, $R$, as a representative dimension of the pit and the rate of its increase, $d R / d t$, as a representative rate of pit growth. Then, from our experimental results given by eqs. (6) and (7) we obtain for the rate of pit growth 


$$
v=\frac{d R}{d t}=\frac{k_{R}^{2}}{2 R}=\frac{k_{s}}{\pi} \cdot \frac{1}{2 R} .
$$

Obviously, this is a parabolic rate equation in which the rate is inversely as the pit dimension, and suggests that the rate is controlled by the diffusion of dissolving metal ion through the pit.

In order to explain the experimental results with respect to the rate constant $k_{R}^{2}\left(=k_{s} / \pi\right)$ and its potential dependence given in Fig. 13, we now postulate a pit model (Fig. 16) in which the inner pit surface has an oxide film forming and breaking down continuously during the pit growth whose thickness is of the same order as of the passivation film; Such a film may be called the transpassive film or electropolishing film. Furthermore, we assume that the ionic diffusion in the solution within the pit is linear under the aiding field $\Delta \phi_{R} / R$ which is relatively small,

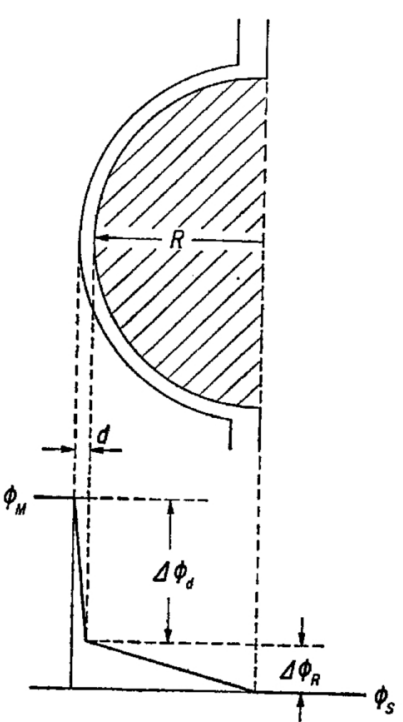

Fig. 16 A proposed model and potential distribution for pit.

$$
v(d)=v_{0}^{\prime} \frac{\Delta \phi_{R}}{R},
$$

while in the surface oxide film non-linear diffusion of the Cabrera and Mott type ${ }^{(27)}$ is limiting the ion migration rate under the very strong aiding field $\Delta \phi_{d} / d$ of the order of $10^{-6} \mathrm{~V} / \mathrm{cm}$,

$$
v(d)=v_{0} \exp \left(\frac{\beta \Delta \phi_{d}}{d}\right),
$$

where $d$ is the thickness of the surface film, $\Delta \phi_{d}$ and $\Delta \phi_{R}$ are the potential difference across the film and in the pit solution, respectively.

At the steady state where $v=v(R)=v(d)$, we obtain from eqs. (19), (20) and (21)

$$
k_{s}=\pi k_{R}^{2}=2 \pi v_{0} R \exp \left(\frac{\beta \Delta \phi_{d}}{d}\right) .
$$

The potential difference $\Delta \phi$ between the metal and the solution outside the pit, to which the electrode potential is directly related, is given by the sum of $\Delta \phi_{d}$ and

(27) N. Cabrera and N.F.Mott : Rep. Progr. in Physics, 12 $(1949), 163$
$\Delta \phi_{R}$. If $\Delta \phi_{d} \gg \Delta \phi_{R}$ as it would be in actual pitting dissolution, $\Delta \phi_{d}$ approximates $\Delta \phi$ and hence eq. (22) gives

$$
k_{s}=2 \pi v_{0} R \exp \left(\frac{\beta \Delta \phi}{d}\right) \text {. }
$$

The pre-exponential factor $\left(2 \pi v_{0} R\right)$ can be assumed to be almost constant compared with the exponential term. Evidently, therefore, this equation explains the experimental fact given in Fig. 13 that $k_{s}$ increases exponentially with the potential holding a Tafel relation.

\section{Conclusion}

(1) The high speed rotation of electrode makes the pit growth very stable and enables the pitting dissolution current from a single pit to be measured. But little does it affect the pitting potential and the pit generation frequency.

(2) By use of rotating electrode it is made possible to measure readily the induction times for successively opening pits and hence the frequency of pit generation at constant potential.

(3) Potentiostatically, pits break out at a constant frequency and hence the number of pits $n$ is a linear function of time $\tau_{n}$,

$$
n=k_{p}\left(\tau_{n}-\tau_{0}\right)
$$

where the rate (or frequency) of pit generation $k_{p}$ increases linearly with the potential.

(4) From the linear dependence of $k_{p}$ on the potential, we may see that the process controlling the pit generation is electrical or electromechanical rather than electrochemical.

(5) An incubation time $\tau_{0}$ is required for the onset of the linear get generation, which obviously differs from the induction time $\tau_{1}$ for opening an initial pit and which may be associated with establishing chloride ion adsorption equilibrium on or in the passivation film.

(6) The pitting dissolution current from a single pit is in proportion to the area of pit mouth, and the current denstiy is constant $\left(i_{0}=8 \mathrm{~A} / \mathrm{cm}^{2}\right)$ irrespective of the potential $(+0.60 \mathrm{~V} \sim+1.00 \mathrm{~V},(\mathrm{SCE}))$, indicating that the mass transfer of dissolving metal ion through the pit mouth is rate limiting. The metal ion concentration in the pit solution, estimated from $i_{0}=8 \mathrm{~A} / \mathrm{cm}^{2}$, is so large $(4 \sim 20 \mathrm{~mol} / \mathrm{L})$ that a polymer of metal chloride or other metal salts can be deposited. We see therefore that the pitting dissolution in the potential range examined is similar to the electropolishing dissolution.

(7) The pit grows following a parabolic law in which the rate decreases inversely as the pit dimension, and the parabolic rate constant increases with the potential obeying a Tafel relation. From this we see that the field-assisting ionic migration through the pit is ratelimiting, and propose a pit model in which a thin barrier layer, continuously forming and breaking down on the inner pit surface, assumes a high electric field; such a layer may be called the transpassive film or electropolishing film.

(8) The form of pits is approximately hemispherical but the ratio of its depth to radius increases as the pit grows. 